\title{
Candida tropicalis is the most prevalent yeast species causing candidemia in Algeria: the urgent need for antifungal stewardship and infection control measures
}

Youcef Megri ${ }^{1+}$, Amir Arastehfar ${ }^{2,3^{*}+}$, Teun Boekhout ${ }^{2,3}$, Farnaz Daneshnia ${ }^{2}$, Caroline Hörtnagl ${ }^{4}$, Bettina Sartori ${ }^{4}$, Ahmed Hafez ${ }^{5}$, Weihua Pan ${ }^{6 *}$, Cornelia Lass-Flörl ${ }^{4}$ and Boussad Hamrioui ${ }^{1}$

\begin{abstract}
Background: Despite being associated with a high mortality and economic burden, data regarding candidemia are scant in Algeria. The aim of this study was to unveil the epidemiology of candidemia in Algeria, evaluate the antifungal susceptibility pattern of causative agents and understand the molecular mechanisms of antifungal resistance where applicable. Furthermore, by performing environmental screening and microsatellite typing we sought to identify the source of infection.

Methods: We performed a retrospective epidemiological-based surveillance study and collected available blood yeast isolates recovered from the seven hospitals in Algiers. To identify the source of infection, we performed environmental screening from the hands of healthcare workers (HCWs) and high touch areas. Species identification was performed by API Auxa-Color and MALDI-TOF MS and ITS sequencing was performed for species not reliably identified by MALDITOF MS. Antifungal susceptibility testing followed CLSI M27-A3/S4 and included all blood and environmental yeast isolates. ERG11 sequencing was performed for azole-resistant Candida isolates. Microsatellite typing was performed for blood and environmental Candida species, where applicable.

\footnotetext{
* Correspondence: a.arastehfar.nl@gmail.com; panweihua@smmu.edu.cn

${ }^{\dagger}$ Youcef Megri and Amir Arastehfar contributed equally to this work.

${ }^{2}$ Yeast Department, Westerdijk Fungal Biodiversity Institute, Utrecht, The Netherlands

${ }^{6}$ Shanghai Key Laboratory Molecular Medical Mycology, Shanghai 200003

China

Full list of author information is available at the end of the article
}

C The Author(s). 2020 Open Access This article is licensed under a Creative Commons Attribution 4.0 International License, which permits use, sharing, adaptation, distribution and reproduction in any medium or format, as long as you give appropriate credit to the original author(s) and the source, provide a link to the Creative Commons licence, and indicate if changes were made. The images or other third party material in this article are included in the article's Creative Commons licence, unless indicated otherwise in a credit line to the material. If material is not included in the article's Creative Commons licence and your intended use is not permitted by statutory regulation or exceeds the permitted use, you will need to obtain permission directly from the copyright holder. To view a copy of this licence, visit http://creativecommons.org/licenses/by/4.0/. The Creative Commons Public Domain Dedication waiver (http://creativecommons.org/publicdomain/zero/1.0/) applies to the data made available in this article, unless otherwise stated in a credit line to the data. 
(Continued from previous page)

Results: Candida tropicalis (19/66) was the main cause of candidemia in these seven hospitals, followed by Candida parapsilosis (18/66), Candida albicans (18/66), and Candida glabrata (7/66). The overall mortality rate was 68.6\% (35/51) and was $81.2 \%$ for C. tropicalis-infected patients (13/16). Fluconazole was the main antifungal drug used (12/51); $41 \%$ of the patients (21/51) did not receive any systemic treatment. Candida parapsilosis was isolated mainly from the hands of HCWs (7/28), and various yeasts were collected from high-touch areas (11/47), including Naganishia albida, C. parapsilosis and C. glabrata. Typing data revealed interhospital transmission on two occasions for C. parapsilosis and C. glabrata, and the same clone of C. parapsilosis infected two patients within the same hospital. Resistance was only noted for C. tropicalis against azoles (6/19) and fluconazole-resistant C. tropicalis isolates ( $\geq 8 \mu \mathrm{g} / \mathrm{ml})(6 / 19)$ contained a novel P56S (5/6) amino acid substitution and a previously reported one (V234F; 1/6) in Erg11p.

Conclusions: Collectively, our data suggest an urgent need for antifungal stewardship and infection control strategies to improve the clinical outcome of Algerian patients with candidemia. The high prevalence of C. tropicalis joined by fluconazole-resistance may hamper the therapeutic efficacy of fluconazole, the frontline antifungal drug used in Algeria.

Keywords: Candidemia, Microsatellite typing, Algeria, Antifungal susceptibility testing, MALDI-TOF MS, ERG11 sequencing, Environmental screening

\section{Introduction}

Bloodstream infections caused by Candida species, i.e., candidemia, are attributable to the annual high rate of mortality worldwide [1] and significant hospital costs of $\$ 1.4$ billion in the US each year [2]. The five most prevalent gut mycobiota constituents, i.e., Candida albicans, Candida tropicalis, Candida parapsilosis, Candida glabrata, and Pichia kudriavzveii (C. krusei) [3] are the major causes of candidemia [4]. Historically, C. albicans is known to be the most prevalent cause of candidemia, but the changing landscape of candidemia epidemiology showed that the prevalence of non-albicans Candida (NAC) species is increasing [4] and in some cases surpassing that of C. albicans [5]. Unfortunately, some of the NAC species, such as $C$. glabrata [6] and Pichia kudriavzveii [7], intrinsically have higher minimum inhibitory concentration (MIC) values toward azoles, and C. glabrata rapidly acquires resistance to echinocandins [6], the frontline antifungal recommended for the treatment of candidemia [8]. Presently, numerous studies in different countries reported the emergence of $C$. parapsilosis [9] and C. tropicalis [10] blood isolates resistant to fluconazole, the frontline antifungal drug used to treat candidemia in developing countries $[5,11]$. Most troubling, the emergence of multidrug-resistant strains of C. glabrata [6] and, more recently, C. auris [12] has led to worrisome therapeutic challenges. Azole resistance mechanisms in $C$. albicans, $C$. parapsilosis, and C. tropicalis is mediated mainly by the occurrence of specific amino acid substitutions in ERG11, resulting in reduced affinity of azoles to the drug target, in addition to overexpression of efflux pumps [7].

Candida species differ in their mode of transmission in the clinical setting. For instance, $C$. albicans candidemia is acquired mostly endogenously [13], while C. parapsilosis is known for being transferred from the hands of healthcare workers (HCWs) resulting in clonal outbreaks in healthcare settings [14]. On the other hand, controversies exist regarding the mode of transmission of $C$. tropicalis, with some believing that it might be horizontally transferred in hospitals [15], while others suggest it is acquired from environmental origins outside of the hospital setting [10]. Regarding C. glabrata, although its infection source is generally endogenous, some studies have found horizontal transfer for this species [16]. As a result, resolutive typing techniques, such as microsatellite typing, are of paramount importance to identify the source of infection [14].

Despite compelling evidence about its importance, a comprehensive study of candidemia in Algeria is lacking. Therefore, we conducted the current study to fill this gap. Yeast isolates collected from 2016 to 2019 from seven hospitals in Algiers were identified and subjected to antifungal susceptibility testing (AFST). The contribution of ERG11 mutations to fluconazole resistance was assessed by ERG11 sequencing of fluconazole-resistant isolates. Environmental screening followed by microsatellite typing was performed to understand the molecular epidemiology of C. parapsilosis, C. tropicalis, and C. glabrata.

\section{Methods}

\section{Settings and study design}

This study was approved by the ethical committee of Mustapha Pasha University Hospital. Yeast isolates collected from 2016 to 2019 regardless of age, sex, underlying conditions, and wards were included in this study. Isolates belonged to seven hospitals in Algiers, namely Mustapha Pacha, Beni Messous, Tizi Ouzou, Parnet, and Blida, EPH Médéa, and EPH Zemirli. Blood isolates were obtained from positive blood bottle cultures incubated in Bactec Device (BD BACTEC ${ }^{\mathrm{T}}$ FX Series, Le Pont-de-Claix, France), from which $100 \mu$ l was transferred onto Sabouraud chloramphenicol agar (SCA) and chromogenic plates (CandiSelect $^{\mathrm{TM}}$ 4, Bio-Rad, Marnes-la-Coquette, France), followed by incubation at $37^{\circ} \mathrm{C}$ for $24-48 \mathrm{~h}$. 
Environmental sampling and identification strategy

Environmental sampling was performed using sterile cotton swabs moistened with sterile normal saline. Forty-seven swab samples were taken from high touch spots and reusable devices and 28 from the hands of HCWs. Swab samples were streaked onto two SDA plates, one containing chloramphenicol and one without, and incubated at $37^{\circ} \mathrm{C}$ for 48-72 h. Plates without growth of yeasts and those with filamentous fungi were excluded from this study. Yeasts were initially identified by API Auxa-Color (Bio-Rad, Marnes-la-Coquette, France) and further characterized by the MALDI Biotyper system (Bruker Daltoniks, Bremen, Germany) using the full-extraction method [17]. Some rare yeast species belonging to the genera of Aureobasidium and Naganishia were further confirmed using internal transcribed spacer ribosomal DNA (ITS rDNA) sequencing via the ITS1 and ITS4 primers [18]. DNA samples were extracted using a CTAB-based buffer and following the suggested protocol [17].

\section{Antifungal susceptibility testing (AFST)}

To determine the MIC values of each species, the broth microdilution method using CLSI-M27/A3 was followed [19]. AFST included the following antifungals; fluconazole (FLZ) (Sigma-Aldrich, St. Louis, MO, USA), voriconazole (VRZ) (Sigma-Aldrich, St. Louis, MO, USA), itraconazole (ITZ) (Sigma-Aldrich, St. Louis, MO, USA) anidulafungin (AND) (Pfizer, NY, USA), micafungin (MFG) (Astellas, Munich, Germany), and amphotericin B (AMB) (SigmaAldrich, St. Louis, MO, USA). MIC values were visually read after $24 \mathrm{~h}$ of incubation at $35^{\circ} \mathrm{C}$, and Pichia kudriavzveii (ATCC 6258) and C. parapsilosis (ATCC 22019) were used for quality control purposes. MIC data were interpreted in a species-specific manner as suggested [20].

\section{ERG11 sequencing}

Candida tropicalis isolates showing fluconazole resistance were subjected to ERG11 sequencing using a defined protocol [21]. The genome of $C$. tropicalis MYA3404 (AAFN00000000.2) was considered the reference wild-type [22]. ERG11 sequences were analysed and curated by SeqMan Pro software (DNASTAR, Madison, WI, USA) and aligned by MEGA software v7.0 [23] in the presence of the wild-type sequence (AAFN00000000.2) (sequences available at the end of the Supplementary files).

\section{Multilocus microsatellite typing}

Environmental and blood C. parapsilosis [24] and C. glabrata [25] isolates and all blood isolates of $C$. tropicalis [26] were subjected to respective multilocus microsatellite typing techniques using published methods [24-26]. Different genotypes were defined when two given strains differed in more than one microsatellite marker tested [24-26]. Microsatellite data were analyzed using Bionumerics software v7.6 (Applied Math, Sint-Martens-Latem, Belgium) and dendrograms were constructed using the unweighted-pair group method by average linkages. Microsatellite data were considered categorical values.

\section{Statistical analysis}

Data included in this study were analyzed using SPSS software v27 (PSS Inc. Chicago, IL, USA).

\section{Availability of sequence data}

ITS sequences of Aureobasidium melanogemum, Naganishia albidus, and Naganishia liquefaciens (MN717161MN717166) and the ERG11 sequences obtained for FLZR C. tropicalis isolates (MN723553-MN723558) were deposited in GenBank (https://www.ncbi.nlm.nih.gov/genbank/).

\section{Results}

\section{Patient characteristics}

In total, 66 yeast isolates were isolated from blood samples of 51 patients (male $(28 / 51 ; 54.9 \%)$, female $(19 / 51 ; 37.2 \%)$ (no data for four patients). Adults $(26 / 51 ; 51 \%)$ and children $(23 / 51 ; 45.1 \%)$ almost equally acquired candidemia (no data for two patients). The vast majority of the patients were hospitalized in Mustapha Pacha $(n=38 / 51 ; 74.5 \%)$, followed by Beni Messous $(n=4 ; 7.8 \%)$ and Tizi Ouzou (each $n=4$; $7.8 \%)$, Parnet $(n=2 ; 3.9 \%)$, and Blida, EPH Médéa, and EPH Zemirli (each $n=1 ; 1.96 \%$ ). Patients were admitted mainly to pediatric $(18 / 51 ; 35.3 \%)$ and ICU wards $(15 / 51 ; 29.4 \%)$, followed by neurology $(5 / 51 ; 9.8 \%)$, gastroenterology $(3 / 51$; $5.8 \%)$, and other wards $(n=10 / 51 ; 19.6)$. Neutropenia $(n=9 /$ $51 ; 17.6 \%)$, leukemia $(n=8 / 51 ; 15.7 \%)$, abdominal surgery and cancer (each $n=4 / 51 ; 7.8 \%$ ) were the most prevalent underlying conditions. Antifungal treatment data were available for only 33 patients (no data for 18 patients), among whom FLZ $(n=12 / 51 ; 23.5 \%)$ and caspofungin $(n=7 / 51$; $13.7 \%)$ were the most widely used systemic antifungals, followed by AMB $(n=3 / 51 ; 5.6 \%)$ (some patients were treated with more than one antifungal); $41 \%$ of the patients $(n=21 / 51)$ did not receive any antifungals. The mortality rate was $68.6 \%(n=35)$ (no data for three patients). The overall mortality rate was $66.6 \%$ (35/51), and per species, patients infected with C. glabrata showed the highest mortality rate (5/6; 83.3\%), followed by $C$. tropicalis (13/16; 81.2\%), C. parapsilosis $(9 / 13 ; 69.2 \%$, no data for one patient), and $C$. albicans $(7 / 11 ; 63.6 \%$, no data for patient). Additionally, the only patient infected with $C$. dubliniensis died. The rest of the patients infected with rare yeasts all survived $(n=3)$.

\section{Identification of yeast isolates and species distributions and prevalence}

Candida tropicalis was the most prevalent species (16 patients, 19 isolates), followed by C. parapsilosis (14 patients, 18 isolates), C. albicans (12 patients, 18 isolates), C. glabrata (6 patients, 7 isolates), Clavispora lusitaniae 
$(n=1)$, Meyerozyma elongisporous $(n=1)$, and Aureobasidium melanogenum $(n=1)$ (Supplementary Table 1$)$. Multiple isolates of the same species were recovered from nine patients as follows: C. albicans $(n=11$ from four patients), C. parapsilosis ( $n=6$ from two patients), C. tropicalis ( $n=5$ from two patients), and C. glabrata ( $n=2$ from one patient). Almost one third of the hands of HCWs (9/28) were positive for yeasts, among which $77.7 \%$ were $C$. parapsilosis $(n=7)$, followed by $C$. orthopsilosis and Prototheca wickerhamii (one isolate each) (Fig. 1, Supplementary Table 1). Approximately $24 \%$ of the high-touch areas were positive for yeasts $(n=11)$, including Naganishia albida (Cryptococcus albidus var. albidus) $(n=3 ; 27.2 \%)$ and $C$. parapsilosis and C. glabrata (each $n=2 ; 18.1 \%$ ) (Fig. 1, Supplementary Table 1). Phylogenetic analysis using the neighbor-joining algorithm and 1000 bootstraps was performed to unequivocally identify isolates of $A$. melanogenum, N. albida, and N. liquefaciens (Supplementary Fig. 1).

\section{Antifungal susceptibility testing}

Candida albicans, C. parapsilosis, and C. glabrata were susceptible to all antifungal drugs tested. Candida dubliniensis, L. elongisporous, and Clavispora lusitaniae showed MIC values lower than ECV toward all antifungal drugs studied (Table 1 and Supplementary Table 2). FLZR was noted for $31.5 \%$ of $C$. tropicalis isolates $(n=6 ; \mathrm{MIC} \geq 8 \mu \mathrm{g} / \mathrm{ml}$ ), and $50 \%$ were cross-resistant to the three azole drugs tested: $83.3 \%$ to FLZ and ITZ $(n=5 ; \mathrm{MIC}>0.5 \mu \mathrm{g} / \mathrm{ml})$, and $66.6 \%$ to FLZ and VRZ ( $n=4 ; \mathrm{MIC} \geq 1 \mu \mathrm{g} / \mathrm{ml})$ (Tables 1 and Supplementary Table 2). Exploring the medical histories of patients infected with fluconazole-resistant (FLZR) isolates showed that three patients received fluconazole (no data for one patient), while one of them did not receive any antifungals in general or azoles in particular during his hospitalization. Isolates from the hands of HCWs were all susceptible to all antifungals tested (Supplementary Table 2). Yeasts isolated from the high touch areas, $N$. albida $(n=2), N$. liquefaciens $(n=1)$, and Rhodotorula mucilaginosa $(n=1)$, showed elevated MIC values for fluconazole $(4-64 \mu \mathrm{g} / \mathrm{ml})$, MFG $(8 \mu \mathrm{g} / \mathrm{ml})$, and AND $(8 \mu \mathrm{g} / \mathrm{ml})$ (Supplementary Table 2).

\section{ERG11 sequencing}

Six C. tropicalis blood isolates resistant to FLZ were subjected to $E R G 11$ sequencing. Isolate \#50 did not carry any nonsynonymous mutations in ERG11, and the remaining of five isolates (\#58, 61-64) carried nonsynonymous mutation of P56S corresponding to the nucleotide mutation C166T. Moreover, isolate \#58 carried an extra nonsynonymous mutation, V234F, corresponding to the nucleotide mutation G700T.

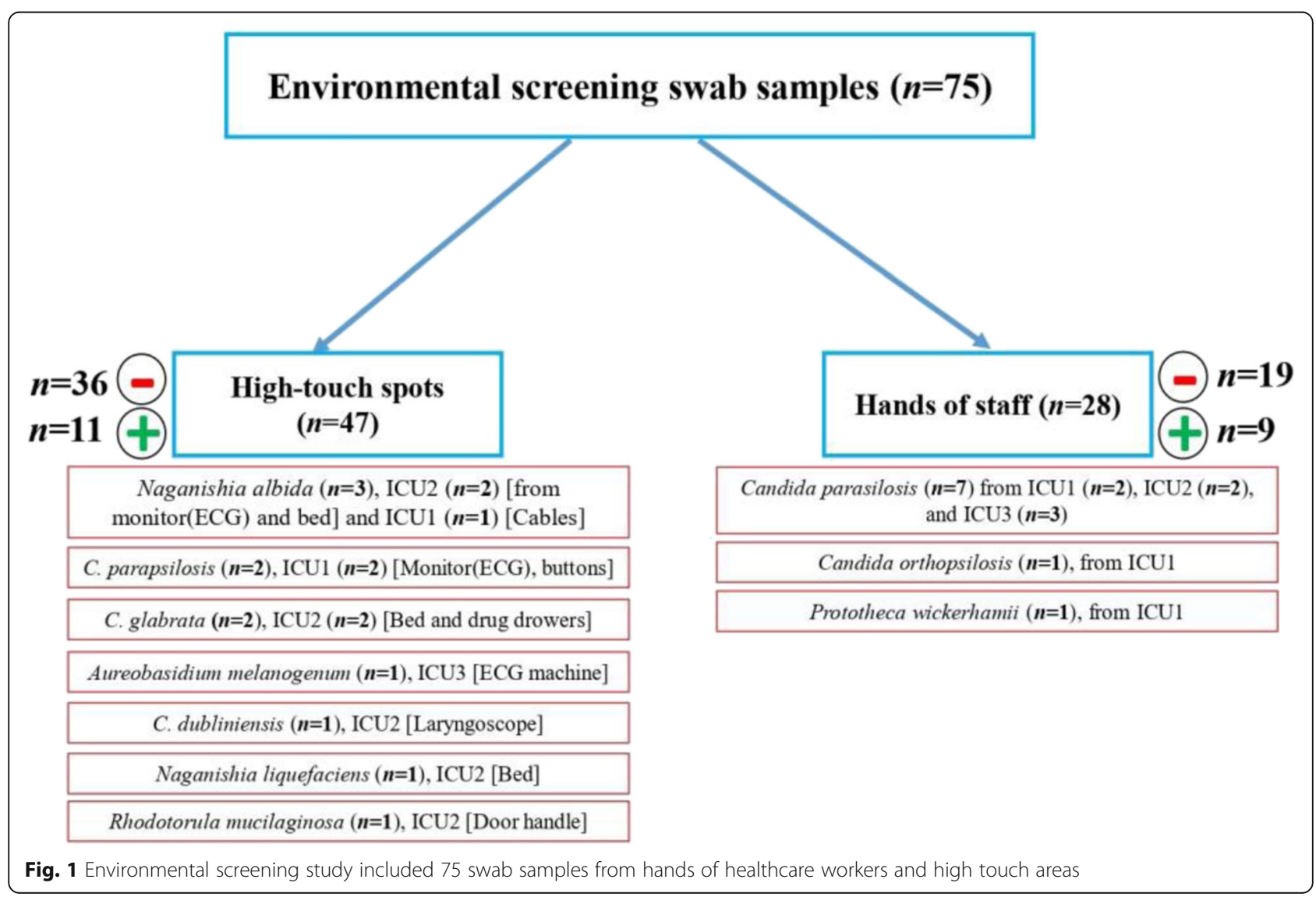


Table 1 Classification of the minimum inhibitory concentration of blood isolates identified in this study based on epidemiological cut-off values and clinical breakpoints

\begin{tabular}{|c|c|c|c|c|c|c|c|}
\hline \multirow[t]{2}{*}{ Species } & \multirow[t]{2}{*}{ Susceptibility } & \multicolumn{6}{|c|}{ MIC values $(\mu \mathrm{g} / \mathrm{ml})$} \\
\hline & & FLZ & VRZ & ITZ & $\mathrm{AMB}$ & MFG & ANF \\
\hline \multirow[t]{4}{*}{ Candida tropicalis $(n=19)$} & $<\mathrm{ECV}$ & 12 & 10 & 13 & 19 & 19 & 19 \\
\hline & $>\mathrm{ECV}$ & 7 & 9 & 5 & 0 & 0 & 0 \\
\hline & $\mathbf{S}$ & 13 & 15 & NA & NA & 19 & 19 \\
\hline & $\mathbf{R}$ & 6 & 4 & NA & NA & 0 & 0 \\
\hline \multirow[t]{4}{*}{ Candida albicans ( $n=18$ ) } & $<\mathrm{ECV}$ & 17 & 17 & 18 & 18 & 18 & 18 \\
\hline & $>\mathrm{ECV}$ & 1 & 1 & 0 & 0 & 0 & 0 \\
\hline & $S$ & 18 & 18 & NA & NA & 18 & 18 \\
\hline & $\mathbf{R}$ & 0 & 0 & NA & NA & 0 & 0 \\
\hline \multirow[t]{4}{*}{ Candida parapsilosis $(n=18)$} & $<\mathrm{ECV}$ & 18 & 18 & 18 & 18 & 18 & 18 \\
\hline & $>\mathrm{ECV}$ & 0 & 0 & 0 & 0 & 0 & 0 \\
\hline & S & 18 & 18 & NA & NA & 18 & 18 \\
\hline & $\mathbf{R}$ & 0 & 0 & NA & NA & 0 & 0 \\
\hline \multirow[t]{4}{*}{ Candida glabrata $(n=7)$} & $<\mathrm{ECV}$ & 7 & 7 & 7 & 7 & 6 & 7 \\
\hline & $>\mathrm{ECV}$ & 0 & 0 & 0 & 0 & 1 & 0 \\
\hline & $\mathrm{S}$ & 7 & NA & NA & NA & 7 & 7 \\
\hline & $\mathbf{R}$ & 0 & NA & NA & NA & 0 & 0 \\
\hline \multirow[t]{2}{*}{ Candida dubliniensis $(n=1)$} & $<\mathrm{ECV}$ & 1 & 1 & 1 & 1 & 1 & 1 \\
\hline & $>\mathrm{ECV}$ & 0 & 0 & 0 & 0 & 0 & 0 \\
\hline \multirow[t]{2}{*}{ Clavispora lusitaniae $(n=1)$} & $<\mathrm{ECV}$ & 1 & 1 & 1 & 1 & 1 & 1 \\
\hline & $>\mathrm{ECV}$ & 0 & 0 & 0 & 0 & 0 & 0 \\
\hline Lodderomyces elongiporous $(n=1)$ & NA & $\leq 0.125$ & $\leq 0.03$ & 0.03 & 0.06 & $\leq 0.0156$ & $\leq 0.0156$ \\
\hline Aureobasidium melanogenum $(n=1)$ & NA & 16 & 0.06 & 0.06 & 0.125 & 1 & 1 \\
\hline
\end{tabular}

ECV Epidemiological cut-off value, $R$ Resistant, $S$ Susceptible, NA Not applicable MIC Minimum inhibitory concentration

\section{Typing analysis}

C. parapsilosis isolates obtained from the hands of HCWs $(n=7)$, ECG monitors and buttons $(n=2)$, and blood $(n=18)$ were subjected to microsatellite typing and revealed 20 genotypes (G1-G20) and six main clusters (C1-C6) (Fig. 2). Among isolates forming defined clusters $(n=21 ; 78 \%) 61.9 \%$ of them $(n=13)$ were identified in intensive care units and $22.7 \%$ in pediatric wards $(n=5)$ (Fig. 2). C6 ( $n=3$, hands; $n=4$, blood) and C2 $(n=3$, hands; $n=1$, blood) contained a mixture of blood and hand and/or ECG monitor origins, while those from C4, C3, and C1 were all obtained from blood (Fig. 2). Clonality was observed only for blood isolates collected from Mustapha Pacha hospital $(n=7)$, among which four isolates formed two distinct clones recovered from two patients in 2019 (isolates \# 15, 16, and 18 from one patient, and isolate \# 10 from another patient, ICU) and the other three isolates (isolates \# 2, 3, and 4, pediatric wards) were from another patient in 2018 (Fig. 2). Interestingly, one of the isolates (\#13) recovered from a blood sample in TiziOuzou hospital shared the same genotype as those obtained from three other blood samples from
Mustapha Pacha hospital (Fig. 2). Candida tropicalis isolates formed six clusters representing 18 genotypes and the vast majority of them were obtained from pediatrics $(n=8 ; 42.1 \%)$ and ICU wards $(n=7 ; 36.8 \%)$ (Fig. 3), among which isolates belonging to C1 (4/4) and $\mathrm{C} 2(2 / 2)$ were from pediatric wards, whereas C6 $(6 / 6)$ was identified in ICU wards. Clonality was observed only for two FLZR isolates obtained from the same patients (\#61 and 62), which were distinct from the first FLZS isolate of the same patient (\#60) (Fig. 3). Regarding Candida glabrata isolates (7 blood and 2 environmental), $57.1 \%$ of the blood isolates (4/7) were recovered from ICU wards (Fig. 4). Candida glabrata isolates showed two clusters $(\mathrm{C} 1, n=2 ; \mathrm{C} 2$, $n=3$ ) (Fig. 4). Surprisingly, one of the C. glabrata isolates in $\mathrm{C} 1$ obtained from a patient bed showed the same genotype as a isolate obtained from a blood sample (Fig. 4). Two patients, one from Mustapha Pacha and one from Beni Messous, were infected with C. glabrata isolates that were $100 \%$ clonal (\#70 and 73 ) and the two isolates from the same patient (\#68 and 73) had the same genotype (Fig. 4). 


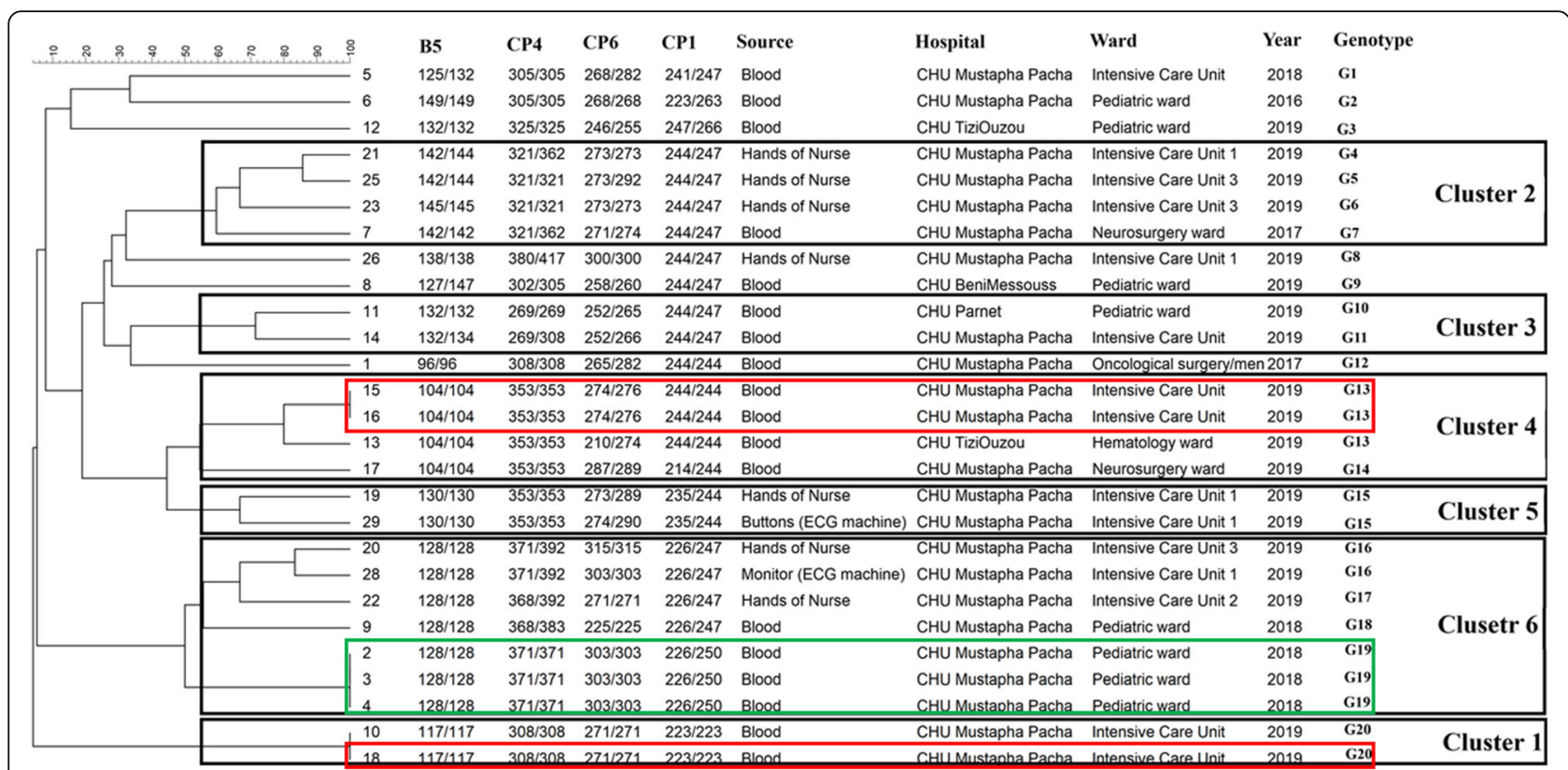

Fig. 2 Microsatellite typing of Candida parapsilosis isolates recovered from environmental screening and blood samples. Rectangular with the same color contained isolates of the same patients

\section{Discussion}

Candida tropicalis with an $81.2 \%$ mortality rate showed the highest rate of FLZ resistance, and microsatellite typing highlighted clusters enriched in ICU and pediatric wards. The high prevalence of $C$. tropicalis together with fluconazole resistance is a serious threat hampering the therapeutic efficacy of fluconazole, the frontline antifungal drug used in Algeria. Typing analysis underscored an ongoing C. parapsilosis outbreak without an obvious source of infection, as well as inter-hospital transmission of $C$. glabrata and C. parapsilosis. A novel amino acid substitution in Erg11p was shown in FLZR C. tropicalis isolates.
In concordance with other studies [5, 27], neutropenia, leukemia, and abdominal surgeries were the most prevalent underlying conditions for our patients. The overall crude mortality rate was high (68.6\%), and patients infected with C. glabrata $(83.3 \%)$ and C. tropicalis (81.2\%) showed the highest rates of mortality. Although insertion of central venous catheter and antibiotic treatment are both prominent risk factors for the development of candidemia, these data were scarce and not well recorded in Algerian hospitals. In line with our findings, a candidemia study in South Korea [28] revealed that patients infected with $C$. tropicalis showed the highest mortality rate (44.1\%) relative to those

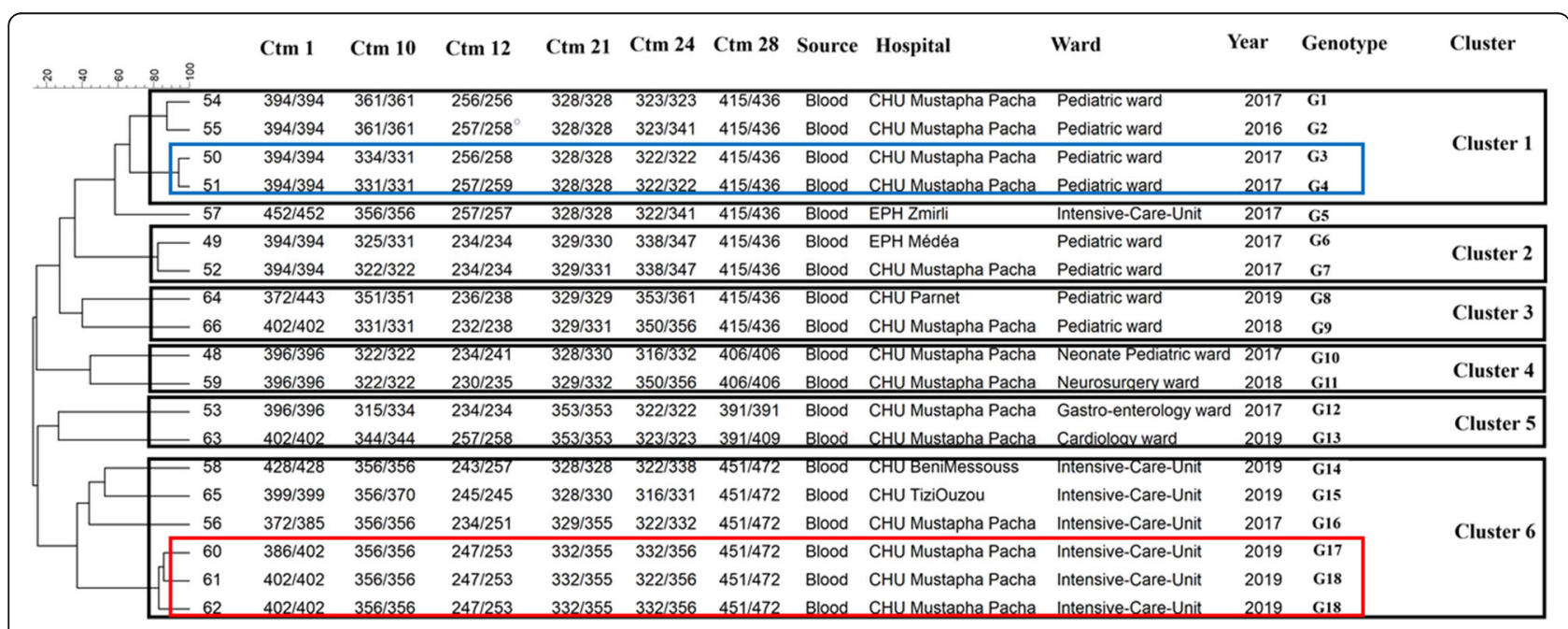

Fig. 3 Microsatellite typing of Candida tropicalis blood isolates. Rectangular with the same color contained isolates of the same patients 


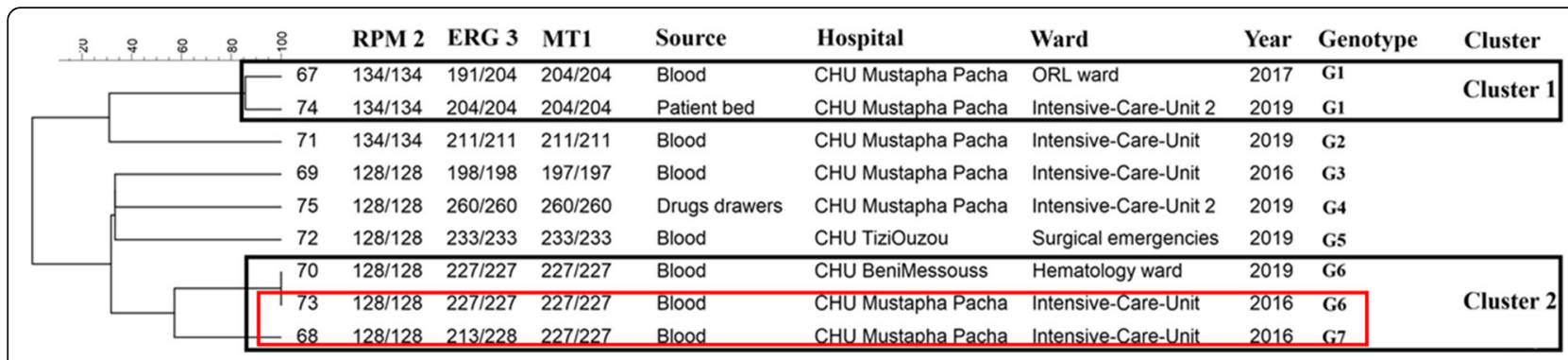

Fig. 4 Microsatellite typing of Candida glabrata isolates recovered from environmental screening and blood samples. Rectangular with the same color contained isolates of the same patients

infected with other non-albicans Candida species. Surprisingly, $44.1 \%$ of the patients did not receive any systemic antifungal treatments and among those treated, FLZ was the most commonly used systemic antimycotic. The low price of FLZ and the high cost of echninocandins are among the factors encouraging medical settings of developing and resource-limited countries to use FLZ for the treatment of the vast majority of candidemia cases $[5,11]$. We found $C$. tropicalis as the most prevalent Candida species, while $C$. albicans ranked third, and C. parapsilosis and C. glabrata were the second and fourth causes of candidemia in Algeria. The predominance of C. tropicalis in Algeria is similar to that in India [5], South Korea [28], and the neighboring country, Tunisia [16]. Moreover, this species is the second cause of candidemia in Brazil [29] and some South East Asian countries [30]. Except for A. melanogenum and C. tropicalis, which showed elevated MIC values/resistance to azoles, all isolates were susceptible or WT to antifungals tested in this study. The lack of antifungal resistance of $C$. glabrata in this study is similar to that seen in Iranian [11] and Indian studies [31], and in contrast to the relatively high rate of fluconazole and echinocandin resistance in the USA [6]. Surprisingly, $31.5 \%$ of the $C$. tropicalis isolates $(n=6)$ were resistant to FLZ, and $50 \%$ of those isolates were cross-resistant to the three azoles tested, with $66.6 \%$ to VRZ and FLZ, and 83.3\% to FLZ and ITZ. Studies in China [32], Taiwan [10], and Denmark [33] observed an alarming increasing trend of azole resistance among $C$. tropicalis isolates. The FLZR $C$. tropicalis isolates were subjected to ERG11 sequencing, and all but one of the isolates harbored nonsynonymous mutations, among which V234F (G700T) has been previously reported for a FLZR $C$. albicans isolate [34], while P56S (C166T) detected in 83.3\% (5/6) of the FLZR isolates was a new mutation. Considering that hydrophobic proline 56 was converted to a polar amino acid of serine (containing a hydroxyl group) and that substitution in neighbor amino acid (A61E) was found solely in FLZR C. albicans isolates [35], P56S may cause FLZR. Heterologous expression analysis of both mutations in a wild-type FLZS C. tropicalis strain is required to clarify their contribution to azole resistance. The high mortality and high fluconazole resistance rate together with the high prevalence of $C$. tropicalis in Algeria, where candidemic patients are treated mainly by FLZ, pose a serious threat for candidemic patients hospitalized in this country.

To gain insights into infection control measures we conducted a comprehensive environmental screening of hightouch areas and hands of HCWs. Candida parapsilosis was the most prevalent yeast species isolated from the hands of HCWs. This result is similar to that in an Italian environmental surveillance study, where C. parapsilosis was the most prevalent yeast isolated from HCW hands [36], but in contrast $C$. tropicalis was identified as a major yeast isolated from the hands of Indian HCWs [30, 37]. Candida parapsilosis blood and hand isolates belonged to 20 different genotypes, but they formed clusters of genetically similar isolates. Moreover, C. parapsilosis isolates obtained from blood samples of two patients were genetically $100 \%$ identical. These findings may indicate a hidden source of $C$. parapsilosis that may have started an outbreak in the ICU of Mustapha Pacha hospital, which was not captured by environmental screening, likely due to the low sensitivity of culture [4]. Isolation of two clonal C. glabrata blood isolates and two C. parapsilosis blood isolates belonging to the same genotype from two hospitals may underscore inter-hospital transmission, likely because some healthcare workers had shifts in both hospitals. Surprisingly, two C. glabrata blood isolates recovered from a patient's bed and blood belonging to the same genotype might be an indication for horizontal transmission of $C$. glabrata, which has been observed in other studies [25]. Although, the lack of isolation of C. tropicalis from environmental sources may reject the horizontal transfer of this species in our study, microsatellite typing showed enrichment of genetically similar clusters in ICU and pediatric wards and we could not explain the phenomenon of FLZR acquisition in an azole-naive patient. We noticed that a primary FLZ-susceptible (FLZS) C. tropicalis isolate from a patient was replaced by FLZR isolates during the course of FLZ treatment, likely due to the selective pressure applied by antifungal treatment [38]. Interestingly, the FLZR C. tropicalis isolates from that patient shared the same genotype but were different from the FLZS one, which could be explained by 
microevolution during resistance development [39]. Of note, genotypic variation weas observed for multiple FLZS isolates recovered from the same patient in this study; therefore, genotypic variation may not always be accompanied by resistance development. Interestingly, the isolate of $C$. orthopsilosis from the hands of a HCW may reinforce the hypothesis that, similar to $C$. parapsilosis, it may have been transferred from the hands of HCWs [40]. Moreover, isolation of A. melanogenum, $N$. albida, and $N$. liquefaciens from high touch areas, which are reported to have elevated MIC values to various antifungals [41-43] and finding Aureobasidium melanogenum in both blood and the environment are worrisome. Findings obtained from environmental screening and microsatellite typing may collectively imply the lack of proper hygiene (both hands and hospital environments) and necessitate the application of effective infection control strategies to eradicate/control fungemia caused by various yeast species. These infection control practices include proper hand hygiene, regular disinfection of hospital environments and high-touch areas, and environmental screening followed by application of typing techniques to identify the source of infection.

The limitations of our study were the retrospective nature of the analysis followed by the lack of additional detailed clinical data and the relatively low numbers of isolates investigated, which is due to underestimation of fungal-related infections in Algeria.

\section{Conclusion}

This study explored the epidemiology of candidemia and the relevant clinical profiles of infected patients in Algeria, for which such data are scant. Moreover, we showed a lack of infection control strategies and antifungal stewardship that should be implemented to improve the patient's' outcomes.

\section{Supplementary information}

Supplementary information accompanies this paper at https://doi.org/10. 1186/s13756-020-00710-z.

Additional file 1: Table S1. Species identification via MALFI-TOF MS, API Auxacolor, and sequencing. Yeast isolates were primarily identified by API Auxacolor and MALDI-TOF MS and the rare yeast isolates were further characterized by sequencing. Table S2. MIC values obtained for the yeast isolates evaluated in the current study. Figure S1. Phylogenetic tree for Aureobasidium melanogenum, Naganishia albida, and Naganishia liquefaciens using neighbor joining algorithm and 1000 bootstraps. Bar shows five nucleotide difference in $100 \mathrm{bps}$.

\footnotetext{
Abbreviations

MALDI-TOF MS: Matrix-assisted laser desorption ionization time-of-flight; ITS rDNA: Internal Transcribed spacer ribosomal DNA; CLSI: Clinical Laboratory Standard Institute; HCW: Healthcare workers; NAC: Non-albicans Candida; MIC: Minim inhibitory concentration; AFST: Antifungal susceptibility testing; SCA: Sabouraud chloramphenicol agar; SDA: Sabouraud dextrose agar; CTAB: Cetyltrimethylammonium bromide; FLZ: Fluconazole; VRZ: Voriconazole; ITZ: Itraconazole; AMB: Amphotericin B; AND: Anidulafungin; MFG: Micafungin; ATCC: American Type Culture Collection; ICU: Intensive care unit; FLZR: Fluconazole-resistant; FLZS: Fluconazole-susceptible; C: Cluster
}

\section{Acknowledgements}

NA.

Authors' contributions

$A A, T B, C L F$, and WP designed the study. AA supervised and coordinated the study. $\mathrm{YM}$ and $\mathrm{BH}$ collected the yeast isolates and clinical data. $\mathrm{BH}$ obtained the ethical approval. YM and BH identified the isolates by Auxa-Color. FD, YM, and AA performed MALDI-TOF MS and sequencing. CLF, BS, and $\mathrm{CH}$ performed antifungal susceptibility testing. WP performed the microsatellite typing, ERG11 sequencing, and financially supported the study. AA analyzed and corrected clinical, microsatellite typing, ERG11 sequencing, and antifungal susceptibility data. AH participated in preparation of microsatellite trees. AA drafted the study and applied revisions from all authors. All authors revised the draft. The author(s) read and approved the final manuscript.

\section{Funding}

This work was supported by National Health Department of China [grant no. 2018ZX10101003]; National Natural Science Foundation of China [grant no. 31770161]; and Shanghai Science and Technology Committee [grants no. 14DZ2272900, 14495800500].

\section{Availability of data and materials}

All data obtained in this study were presented in the form of tables, figure, and supplementary data. GenBank data obtained for sequencing of genes of interest are included in this study.

\section{Ethics approval and consent to participate}

This study was approved by ethical committee of Mustapha Pasha University Hospital. Patient's identity were anonymized through assigning numerical codes. Due to the retrospective nature of the study, consent forms were not applicable to this study.

\section{Consent for publication}

NA.

\section{Competing interests}

Authors declared that this study was conducted in absence of any financial relationship that could be considered as a potential conflict of interest.

\section{Author details}

1Parasitology and Mycology Department, Mustapha University Hospital, 16000 Algiers, Algeria. ${ }^{2}$ Yeast Department, Westerdijk Fungal Biodiversity Institute, Utrecht, The Netherlands. Institute of Biodiversity and Ecosystem Dynamics, University of Amsterdam, Amsterdam, the Netherlands. ${ }^{4}$ Institute of Hygiene and Medical Microbiology, Medical University of Innsbruck, Innsbruck, Austria. ${ }^{5}$ Biotechvana, 46980 Paterna, Valencia, Spain. ${ }^{6}$ Shanghai Key Laboratory Molecular Medical Mycology, Shanghai 200003, China.

Received: 2 January 2020 Accepted: 24 March 2020

Published online: 07 April 2020

\section{References}

1. Brown GD, Denning DW, Gow NAR, Levitz SM, Netea MG, White TC. Hidden killers: human fungal infections. Sci Transl Med. 2012;4:165rv13.

2. Benedict $K$, Jackson BR, Chiller T, Beer KD. Estimation of direct healthcare costs of fungal diseases in the United States. Clin Infect Dis. 2019;68:1791-7.

3. Hallen-Adams HE, Suhr MJ. Fungi in the healthy human gastrointestinal tract. Virulence. 2017:8:352-8

4. Lamoth F, Lockhart SR, Berkow EL, Calandra T. Changes in the epidemiological landscape of invasive candidiasis. J Antimicrob Chemother. 2018;73:i4-i13.

5. Chakrabarti A, Sood P, Rudramurthy SM, Chen S, Kaur H, Capoor M, Chhina D, Rao R, Eshwara VK, Xess I, Kindo AJ, Umabala P, Savio J, Patel A, Ray U, Mohan S, Iyer R, Chander J, Arora A, Sardana R, Roy I, Appalaraju B, Sharma A, Shetty A, Khanna N, Marak R, Biswas S, Das S, Harish BN, Joshi S, Mendiratta D. Incidence, characteristics and outcome of ICU-acquired candidemia in India. Intensive Care Med. 2014;41:285-95.

6. Healey KR, Perlin DS. Fungal resistance to Echinocandins and the MDR phenomenon in Candida glabrata. Journal of fungi. 2018:4:E105.

7. Berkow EL, Lockhart SR. Fluconazole resistance in Candida species: a current perspective. Infec Drug Resist. 2017;10:237-45. 
8. Pappas PG, Kauffman CA, Andes DR, Clancy CJ, Marr KA, Ostrosky-Zeichner L, Reboli AC, Schuster MG, Vazquez JA, Walsh TJ, Zaoutis TE, Sobel JD. Clinical practice guideline for the Management of Candidiasis: 2016 update by the Infectious Diseases Society of America. Clin Infect Dis. 2016;62:e1-50.

9. Choi YJ, Kim Y-J, Yong D, Byun J-H, Kim TS, Chang YS, Choi MJ, Byeon SA, Won EJ, Kim SH, Shin MG, Shin JH. Fluconazole-resistant Candida parapsilosis bloodstream isolates with Y132F mutation in ERG11 gene, South Korea. Emerg Infect Dis. 2018;24:1768-70.

10. Chen P-Y, Chuang Y-C, Wu U-I, Sun H-Y, Wang J-T, Sheng W-H, Lo H-J, Wang H-Y, Chen Y-C, S-CC. Clonality of fluconazole-nonsusceptible Candida tropicalis in bloodstream infections, Taiwan, 2011-2017. Emerg Infect Dis. 2019;25:1668-75.

11. Arastehfar A, Daneshnia F, Zomorodian K, Najafzadeh M-J, Khodavaisy S, Zarrinfar H, Hagen F, Shahrabadi ZZ, Lackner M, Mirhendi H, Salehi M, Roudbary M, Pan W, Kostrzewa M, Boekhout T. Low level of antifungal resistance in Iranian isolates of Candida glabrata recovered from blood samples from multicenter (2015-2018): potential prognostic values of genotyping and sequencing of PDR1. Antimicrob Agents Chemother. 2019; 63(7):e02503-18.

12. Jeffery-Smith A, Taori SK, Schelenz S, Jeffery K, Johnson EM, Borman A, Manuel R, Brown CS. Candida auris: a review of the literature. Clin Microbiol Rev. 2018:31:e00029-17.

13. Mayer FL, Wilson D, Hube B. Candida albicans pathogenicity mechanisms. Virulence. 2013;4:119-28.

14. Delfino D, Scordino F, Pernice I, Lo Passo C, Galbo R, David A, Barberi I, Criseo G, Cascio A, Romeo O. Potential association of specific Candida parapsilosis genotypes, bloodstream infections and colonization of health workers' hands. Clin Microbiol Infect. 2014;20:0946-51.

15. Scordino F, Giuffrè L, Barberi G, Merlo FM, Orlando MG, Giosa D, Romeo O. Multilocus sequence typing reveals a new cluster of closely related Candida tropicalis genotypes in Italian patients with neurological disorders. Front Microbiol. 2018;9:1-10.

16. Abbes S, Sellami H, Sellami A, Makni F, Mahfoudh N, Makni H, Khaled S, Ayadi A. Microsatellite analysis and susceptibility to FCZ of Candida glabrata invasive isolates in Sfax hospital, Tunisia. Med Mycol. 2010;49:10-5.

17. Arastehfar A, Fang W, Pan W, Liao W, Yan L, Boekhout T. Identification of nine cryptic species of Candida albicans, C. glabrata, and C. parapsilosis complexes using one-step multiplex PCR. BMC Infect Dis. 2018;18:480.

18. Stielow JB, Lévesque CA, Seifert KA, Meyer W, Irinyi L, Smits D, Renfurm $R$, Verkley GJM, Groenewald M, Chaduli D, Lomascolo A, Welti S, LesageMeessen L, Favel A, Al-Hatmi AMS, Damm U, Yilmaz N, Houbraken J, Lombard L, Quaedvlieg W, Binder M, Vaas LAI, Vu D, Yurkov A, Begerow D, Roehl O, Guerreiro M, Fonseca A, Samerpitak K, van Diepeningen AD, Dolatabadi S, Moreno LF, Casaregola S, Mallet S, Jacques N, Roscini L, Egidi E, Bizet C, Garcia-Hermoso D, Martín MP, Deng S, Groenewald JZ, Boekhout T, de Beer ZW, Barnes I, Duong TA, Wingfield MJ, de Hoog GS, Crous PW, Lewis CT, Hambleton S, Moussa TAA, Al-Zahrani HS, Almaghrabi OA, LouisSeize G, Assabgui R, McCormick W, Omer G, Dukik K, Cardinali G, Eberhardt U, de Vries M, Robert V. One fungus, which genes? Development and assessment of universal primers for potential secondary fungal DNA barcodes. Persoonia. 2015;35:242-63.

19. Clinical and Laboratory Standards Institute. Reference Method for Broth Dilution Antifungal Susceptibility Testing of Yeasts; Approved Standard. 3rd ed. Wayne: M27-A3. CLSl; 2008.

20. Pfaller MA, Diekema DJ. Progress in antifungal susceptibility testing of Candida spp. by use of clinical and laboratory standards institute broth microdilution. J Clin Microbiol. 2012;50:2846-56.

21. Arastehfar A, Daneshnia F, Hafez A, Khodavaisy S, Najafzadeh M-J, Charsizadeh A, Zarrinfar H, Salehi M, Shahrabadi ZZ, Sassani E, Zomorodian K, Pan W, Hagen F, Macit Ilkit MK, TB. Antifungal susceptibility, genotyping, resistance mechanism, and clinical profile of Candida tropicalis blood isolates. Med Mycol. 2020. https://doi.org/10.1093/mmy/myz124.

22. Butler G, Rasmussen MD, Lin MF, Santos MAS, Sakthikumar S, Munro CA, Rheinbay E, Grabherr M, Forche A, Reedy JL, Agrafioti I, Arnaud MB, Bates S, Brown AJP, Brunke S, Costanzo MC, Fitzpatrick DA, de Groot PWJ, Harris D, Hoyer LL, Hube B, Klis FM, Kodira C, Lennard N, Logue ME, Martin R, Neiman AM, Nikolaou E, Quail MA, Quinn J, Santos MC, Schmitzberger FF, Sherlock G, Shah P, Silverstein KAT, Skrzypek MS, Soll D, Staggs R, Stansfield I, Stumpf MPH, Sudbery PE, Srikantha T, Zeng Q, Berman J, Berriman M, Heitman J, Gow NAR, Lorenz MC, Birren BW, Kellis M, Cuomo CA. Evolution of pathogenicity and sexual reproduction in eight Candida genomes. Nature. 2009;459:657-62.
23. Kumar S, Stecher GTK. Molecular evolutionary genetics analysis version 7.0 for bigger datasets. Mol Biol Evol. 2016;33:1870-4.

24. Trobajo-Sanmartín C, Ezpeleta G, Pais C, Eraso E, Quindós G. Design and validation of a multiplex PCR protocol for microsatellite typing of Candida parapsilosis sensu stricto isolates. BMC Genomics. 2018;19:1-10.

25. Foulet F, Nicolas N, Eloy O, Botterel F, Gantier JC, Costa JM, Bretagne S. Microsatellite marker analysis as a typing system for Candida glabrata. J Clin Microbiol. 2005;43:4574-9.

26. Wu Y, Zhou H, Che J, Li W, Bian F, Yu S, Zhang L, Lu J. Multilocus microsatellite markers for molecular typing of Candida tropicalis isolates. BMC Microbiol. 2014;14:245.

27. Cleveland AA, Farley MM, Harrison LH, Stein B, Hollick R, Lockhart SR, Magill SS, Derado G, Park BJ, Chiller TM. Changes in incidence and antifungal drug resistance in candidemia: results from population-based laboratory surveillance in Atlanta and Baltimore, 2008-2011. Clin Infect Dis. 2012;55:1352-61.

28. Ko J-H, Jung DS, Lee JY, Kim HA, Ryu SY, Jung S-I, Joo E-J, Cheon S, Kim Y-S, Kim S-W, Cho SY, Kang C-I, Chung DR, Lee NY, Peck KR. Poor prognosis of Candida tropicalis among non-albicans candidemia: a retrospective multicenter cohort study, Korea. Diagn Microbiol Infect Dis. 2019;95(2):195-200.

29. Wille MP, Guimaraes T, Furtado GHC, Colombo AL. Historical trends in the epidemiology of candidaemia: analysis of an 11-year period in a tertiary care hospital in Brazil. Mem Inst Oswaldo Cruz. 2013;108(3). https://doi.org/ 10.1590/S0074-02762013000300005.

30. Tan BH, Chakrabarti A, Li RY, Patel AK, Watcharananan SP, Liu Z, Chindamporn A, Tan AL, Sun PL, Wu UI, Chen YC, Xu YC, Wang H, Sun ZY, Wang LL, Lu J, Yang Q, Zhang QQ, Shao HF, Liao K, Woo PCY, Marak RSK, Kindo AJ, Wu CL, Ho MW, Lu PL, Wang LS, Riengchan P. Incidence and species distribution of candidaemia in Asia: a laboratory-based surveillance study. Clin Microbiol Infect. 2015;21:946-53.

31. Singh A, Healey KR, Yadav P, Upadhyaya G, Sachdeva N, Sarma S, Kumar A, Tarai B, Perlin DS, Chowdhary A. Absence of azole or echinocandin resistance in candida glabrata isolates in India despite background prevalence of strains with defects in the dna mismatch repair pathway. Antimicrob Agents Chemother. 2018:62:1-9.

32. Fan X, Xiao M, Liao K, Kudinha T, Wang H, Zhang L, Hou X, Kong F, Xu Y-C. Notable Increasing Trend in Azole Non-susceptible Candida tropicalis Causing Invasive Candidiasis in China (August 2009 to July 2014): Molecular epidemiology and clinical azole consumption. Front Microbiol. 2017;8:464.

33. Arendrup MC, Bruun B, Christensen JJ, Fuursted K, Johansen HK, Kjaeldgaard P, Knudsen JD, Kristensen L, Moller J, Nielsen L, Rosenvinge FS, Roder B, Schonheyder HC, Thomsen MK, Truberg K. National surveillance of fungemia in Denmark (2004 to 2009). J Clin Microbiol. 2011;49:325-34.

34. Feng W, Yang J, Xi Z, Qiao Z, Lv Y, Wang Y, Ma Y, Wang Y, Cen W. Mutations and/or Overexpressions of ERG4 and ERG11 genes in clinical azoles-resistant isolates of Candida albicans. Microb Drug Resist. 2017;23:563-70.

35. Jensen RH, Astvad KMT, Silva LV, Sanglard D, Jørgensen R, Nielsen KF, Mathiasen EG, Doroudian G, Perlin DS, Arendrup MC. Stepwise emergence of azole, echinocandin and amphotericin B multidrug resistance in vivo in Candida albicans orchestrated by multiple genetic alterations. J Antimicrob Chemother. 2015;70:2551-5.

36. Sabino R, Sampaio P, Rosado L, Videira Z, Grenouillet F, Pais C. Analysis of clinical and environmental Candida parapsilosis isolates by microsatellite genotyping-a tool for hospital infection surveillance. Clin Microbiol Infect. 2015;21:954.e1-8.

37. Chakrabarti A, Chatterjee SS, Rao KLN, Zameer MM, Shivaprakash MR, Singhi $S$, Singh R, Varma SC. Recent experience with fungaemia: change in species distribution and azole resistance. Scand J Infect Dis. 2009:41:275-84.

38. Lewis JS 2nd, Wiederhold NP, Wickes BL, Patterson TF, Jorgensen JH. Rapid emergence of echinocandin resistance in Candida glabrata resulting in clinical and microbiologic failure. Antimicrob Agents Chemother. 2013;57:4559-61.

39. Healey KR, Jimenez Ortigosa C, Shor E, Perlin DS. Genetic drivers of multidrug resistance in Candida glabrata. Front Microbiol. 2016;7:1995.

40. Arastehfar A, Khodavaisy S, Daneshnia F, Najafzadeh M-J, Mahmoudi S, Charsizadeh A, Salehi M-R, Zarrinfar H, Raeisabadi A, Dolatabadi S, Zare Shahrabadi Z, Zomorodian K, Pan W, Hagen F, Boekhout T. Molecular Identification, Genotypic Diversity, Antifungal Susceptibility, and Clinical Outcomes of Infections Caused by Clinically Underrated Yeasts, Candida orthopsilosis, and Candida metapsilosis: An Iranian Multicenter Study (20142019). Front Cell Infect Microbiol. 2019;9:264.

41. Conde-Pereira C, Rodas-Rodríguez L, Díaz-Paz M, Palacios-Rivera H, Firacative C, Meyer W, Alcázar-Castillo M. Fatal case of polymicrobial meningitis 
caused by Cryptococcus liquefaciens and mycobacterium tuberculosis complex in a human immunodeficiency virus-infected patient. J Clin Microbiol. 2015;53:2753-5.

42. Najafzadeh MJ, Sutton DA, Keisari MS, Zarrinfar H, De Hoog GS, Chowdhary A, Meis JF. In vitro activities of eight antifungal drugs against 104 environmental and clinical isolates of Aureobasidium pullulans. Antimicrob Agents Chemother. 2014;58:5629-31.

43. Choe YJ, Blatt DB, Yalcindag A, Geffert SF, Bobenchik AM, Michelow IC. Cryptococcus albidus Fungemia in an immunosuppressed child: case report and systematic literature review. J Pediatr Infect Dis Soc. 2019;9(1):100-5.

\section{Publisher's Note}

Springer Nature remains neutral with regard to jurisdictional claims in published maps and institutional affiliations.

Ready to submit your research? Choose BMC and benefit from:

- fast, convenient online submission

- thorough peer review by experienced researchers in your field

- rapid publication on acceptance

- support for research data, including large and complex data types

- gold Open Access which fosters wider collaboration and increased citations

- maximum visibility for your research: over $100 \mathrm{M}$ website views per year

At BMC, research is always in progress.

Learn more biomedcentral.com/submissions 